\title{
Manifestações orais da sífilis: revisão sistematizada de literatura
}

\author{
Oral manifetations of syphilis: systematized review of literature \\ Manifestaciones orales de sífilis: revisión sistematizada de literatura \\ Erison Santana dos SANTOS \\ Jamile de Oliveira SÁ \\ Rachel LAMARCK
}

Departamento de Diagnóstico Oral, Faculdade de Odontologia de Piracicaba, UNICAMP Universidade Estadual de Campinas, 13414-903, Piracicaba, São Paulo -SP, Brasil

\begin{abstract}
Resumo
A sífilis é uma infecção bacteriana exclusiva de seres humanos que é transmitida principalmente através de relações sexuais sem o uso de preservativos. Embora seja mais comum em regiões genitais, pode manifestar sinais e sintomas na cavidade oral e mimetizar lesões potencialmente malignas e, dependendo do seu estágio, pode levar ao óbito. O objetivo desse estudo foi realizar uma revisão de literatura a fim de conhecer as principais manifestações orais da sífilis. Foi realizado um levantamento bibliográfico na base de dados PubMed e SciELO. A busca foi realizada durante o mês de agosto e setembro de 2018. No PubMed, foram utilizados os termos "syphilis oral", "manifestations of syphilis", "syphilis in oral cavity", "syphilis in buccal cavity", "syphilis in mouth". Na SciELO, foram usadas os descritores: "oral manifestations of syphilis", "manifestações orais AND sífilis". As manifestações orais da sífilis embora sejam raras, são importantes para o cirurgiã-dentista diagnosticar a infecção em estágios menos agressivos da doença. Pode manifestar-se de diversas formas, dependendo do período de evolução da infecção. Sua incidência vem aumentando devido às mudanças de hábitos na população, principalmente nos grupos de risco. Sendo assim, conhecer as principais manifestações orais da sífilis é importante para intervir em estágios menos avançados, visto que é uma infecção que pode se disseminar rapidamente e levar o paciente a óbito se não tratada.
\end{abstract}

Descritores: Sífilis; Manifestações Bucais; Cancro; Treponema pallidum.

\section{Abstract}

Syphilis is a bacterial infection, caused by Treponema Pallidum, exclusively of humans that is transmitted primarily through sexual intercourse without the use of condoms. Although it is more common in genital regions, it can manifest signs and symptoms in the oral cavity and mimic potentially malignant lesions and, depending on its stage, can cause death. The aim of this study was to perform a literature review in order to know the main oral manifestations of syphilis. A bibliographic survey was carried out in the PubMed and SciELO database. The search was performed during the month of August and September 2018. In PubMed, the terms "oral syphilis", "manifestations of syphilis", "syphilis in oral cavity", "syphilis in buccal cavity", "syphilis in mouth ". In SciELO, the key words used were: "oral manifestations of syphilis", "oral manifestations AND syphilis". Although oral manifestations of syphilis are rare, it is important for the dentist to diagnose the infection in less aggressive stages of the disease. It can manifest itself in several ways, depending on the period of evolution of the infection. Its incidence is increasing due to the changes of habits in the population, mainly in the groups of risk. Therefore, knowing the main oral manifestations of syphilis is important to intervene in less advanced stages, since it is an infection that can spread rapidly and lead the patient to death if left untreated.

Descriptors: Syphilis; Oral Manifestations; Chancre; Treponema pallidum.

\section{Resumen}

La sífilis es una infección bacteriana exclusiva de seres humanos que se transmite principalmente a través de relaciones sexuales sin el uso de preservativos. Aunque es más común en regiones genitales, puede manifestar signos y síntomas en la cavidad oral y mimetizar lesiones potencialmente malignas y, dependiendo de su etapa, puede llevar a la muerte. El objetivo de este estudio fue realizar una revisión de literatura a fin de conocer las principales manifestaciones orales de la sífilis. Se realizó un levantamiento bibliográfico en la base de datos PubMed y SciELO. La búsqueda fue realizada durante el mes de agosto y septiembre de 2018. En el PubMed, se utilizaron los términos "syphilis oral", "manifestaciones de syphilis", "syphilis in oral cavity", "syphilis in buccal cavity", "syphilis in en boca ". En la SciELO, se utilizaron las palabras clave: "oral manifestations of syphilis", "manifestaciones orales AND sífilis". Las manifestaciones orales de la sífilis aunque son raras, son importantes para el cirujano dentista diagnosticar la infección en etapas menos agresivas de la enfermedad. Puede manifestarse de diversas formas, dependiendo del período de evolución de la infección. Su incidencia viene aumentando debido a los cambios de hábitos en la población, principalmente en los grupos de riesgo. Por lo tanto, conocer las principales manifestaciones orales de la sífilis es importante para intervenir en etapas menos avanzadas, ya que es una infección que puede diseminarse rápidamente y llevar al paciente a muerte si no se trata.

Descriptores: Sífilis; Manifestaciones Bucales; Chancro; Treponema pallidum.

\section{INTRODUÇÃO}

A sífilis é uma doença infecciosa causada pela bactéria espiroqueta Treponema pallidum, subespécie Pallidu ${ }^{1}$. A infecção pode ser adquirida por meio do contato direto com lesões altamente infectantes, na maioria dos casos durante as relações sexuais $^{2}$. Clinicamente a sífilis é caracterizada por vários estágios clínicos e envolvimento simultâneo de diferentes órgãos e tecidos, incluindo a mucosa bucal $^{3}$. De acordo com a evolução temporal, a infecção é classificada em primária, secundária, latente e terciária ${ }^{2}$. Ainda existe a sífilis congênita, que ocorre quando o treponema é transmitido da mãe infectada para o feto por via transplacentária ${ }^{2,4}$.

Estimativas epidemiológicas globais evidenciaram diminuição na incidência da sífilis após a segunda guerra mundial, especialmente em países desenvolvidos. Entretanto, dados atuais demonstram que as taxas de infecção continuam elevadas entre populações dos países de alta renda e permanecem em níveis endêmicos naqueles de baixa e média renda $^{5}$. A cada ano estima-se que haja, a nível mundial, seis milhões de novos casos de sífilis em pessoas entre 15 e 49 anos de idade, sendo que mais de 300.000 mortes fetais e neonatais são atribuídas à sífilis ${ }^{6}$.

As manifestações orais da sífilis ocorrem especialmente no estágio secundário da doença e o aspecto clínico das lesões pode ser bastante heterogêneo e até mimetizar algumas lesões orais potencialmente malignas, como a leucoplasia oral e até mesmo o carcinoma de células escamosas ${ }^{2,4}$. Portanto, o cirurgião-dentista tem um papel 
importante no diagnóstico desta doença, pois a cavidade bucal representa um dos sítios extragenitais mais comuns de inoculação da bactéria ${ }^{4,6}$.

Sendo assim, o objetivo desse trabalho é realizar um levantamento bibliográfico nas bases de dados PubMed e SciELO descrever as principais manifestações orais dos diferentes estágios da sífilis e tornar o diagnóstico mais preciso, especialmente em estágios iniciais, facilitando, portanto, o direcionamento para o tratamento mais adequado.

\section{MATERIAL E MÉTODO}

Para composição desse estudo, foi realizado um levantamento nas bases de dados PubMed - U.S. National Library of Medicine e SciELO - Scientific Eletronic Librari Online. No PubMed, foram utilizados os termos "syphilis oral", "manifestations of syphilis", "syphilis in oral cavity", "syphilis in buccal cavity", "syphilis in mouth". Na base de dados SciELO, foram usadas as palavras-chaves: "oral manifestations of syphilis", "manifestações orais AND sífilis". Esta revisão constitui-se em três etapas. A primeira foi caracterizada pela busca entre os meses de agosto e setembro de 2018 dos artigos nas bases de dados, com a seleção dos artigos científicos sobre o tema. A segunda etapa foi a leitura dos títulos e resumos, com intuito de maior aproximação do tema estudado. Nessa etapa, os artigos que estavam dentro dos critérios de inclusão foram selecionados para a etapa seguinte. Os critérios de inclusão foram: artigos publicados em inglês, português e espanhol, com relevância para o tema, publicados entre os anos de 2000 a 2018. Artigos que não se encontravam publicados na integra, em outros idiomas e publicados antes do ano 2000 foram excluídos da revisão. Na terceira etapa, os artigos foram lidos na integra e incluídos na revisão de literatura. Na leitura e avaliação, os artigos que apresentaram os critérios de elegibilidade foram selecionados e incluídos na pesquisa por consenso entre os autores. Um livro foi adicionado por ser contributivo ao tema.

\section{RESULTADOS}

Após as buscas pelos artigos nas bases de dados foram selecionados 16 artigos e 1 livro com maior contribuição para a pesquisa e que atendiam aos critérios de inclusão e exclusão (Quadro 1).

\section{DISCUSSÃO}

A sífilis é uma doença sistêmica sexualmente transmissível causada pela espiroqueta anaeróbia Treponema Pallidum, que afeta apenas humanos e pode invadir praticamente qualquer órgão ${ }^{1}$. Essa infecção era comum no século passado, porém, em 1940 com a introdução da penicilina tornou-se menos comum $^{1,2}$. Entretanto, tem-se observado um aumento da sua incidência desde 1980 devido principalmente a imunossupressão, aumento na frequência de infecção pelo HIV, negligências no uso de preservativos durante as relações sexuais, além do alto números de parceiros sexuais ${ }^{4}$.

Em geral, os homens são mais afetados pela sífilis do que as mulheres e este perfil pode ser explicado pelo maior risco da doença em homens que fazem sexo com homens ${ }^{4}$. Os pacientes geralmente são adultos em sua terceira a sexta décadas de vida, e a idade média dos homens é maior que a das mulheres ${ }^{4}$. Uma forte associação entre sífilis e infecção pelo Vírus da Imunodeficiência Humana (HIV) pode ser observado. Os pacientes com Infecções Sexualmente Transmissíveis (IST's) podem apresentar risco mais elevado de contaminação pelo $\mathrm{HIV}^{2}$. Esta associação não é incomum, principalmente entre homens que fazem sexo com outros homens e costuma ser mais agressiva que essas lesões isoladas ${ }^{2}$.

- Manifestações orais da sífilis primária

A sífilis primária é caracterizada pela penetração do $T$. pallidum com a formação de cancro duro e uma linfadenomegalia reativa associada $^{2}$. O período de incubação pode variar de 3 dias a 3 meses ${ }^{1,2,4,12}$. As manifestações bucais nessa fase são pouco frequentes, mas o cancro oral manifesta-se como uma úlcera inespecífica, indolor, autolimitada que afeta principalmente a língua, os lábios e a comissura ${ }^{12}$. O lábio superior é mais acometido em homens e o inferior em mulheres, provavelmente devido aos hábitos de felação e cunilíngua $^{4,1}$. Caso a infecção não seja tratada pode evoluir e apresentar outros estágios de manifestações ${ }^{6}$.

- Manifestações bucais da sífilis secundária

Este estágio está associado à disseminação hematogência do microrganismo e os sinais e sintomas são mais frequentes a partir da quarta semana da infecção inicial, podendo surgir antes da resolução completa da sífilis primária ${ }^{4,6}$. Os pacientes geralmente apresentam sintomas sistêmicos como fadiga, mal-estar, febre, dor de garganta, perda de peso, dor de cabeça e linfadenomegalia reativa ${ }^{7}$. Lesões de pele, na forma de uma erupção autolimitada simétrica com predileção para o tronco e extremidades, podem ser vistas ${ }^{7,9,11^{3}}$.

As manifestações orais mais comuns associadas à sífilis secundária são manchas mucosas esbranquiçadas ou avermelhadas, cobertas por uma pseudomembrana fibrinosa ${ }^{2,4,15}$. Pode-se notar a presença de condilomas lata, ulcerações em forma de caracol, maculas papulares vermelhas e pápulas fendidas ${ }^{4,5,8,10,14}$. Nesse estágio a doença é altamente contagiosa $^{13,16,17}$. A apresentação clínica da sífilis secundária torna o diagnóstico diferencial desafiador e amplo, incluindo as úlceras traumáticas, infecções herpéticas, tuberculose, líquen plano, eritema multiforme e carcinoma de células escamosas ${ }^{2}$. Se não diagnosticada nesta fase, as lesões podem 
desaparecer espontaneamente e os pacientes podem entrar em uma fase latente sem sinais da doença ${ }^{1,13}$, com duração de até 30 anos, podendo ou não evoluir para a sífilis terciaria ${ }^{2}$. Aproximadamente $30 \%$ dos pacientes evoluem para a sífilis terciaria ${ }^{4}$.

\begin{tabular}{|l|l|}
\hline Autor/Ano & Título \\
\hline Leuci et al. 2013 & $\begin{array}{l}\text { Oral Syphilis: a retrospective analysis of 12 cases and a } \\
\text { review of literature }\end{array}$ \\
\hline Leão et al. 2006 & Oral manifestations of syphilis \\
\hline Bruce et al. 2004 & Oral manifestations of sexually transmitted diseases \\
\hline Neville et al. 2016 & Patologia Oral e Maxilofacial \\
\hline Kojima et al. 2018 & An Update on the Global Epidemiology of Syphilis \\
\hline WHO, 2016 & $\begin{array}{l}\text { Global health sector strategy on sexually transmitted } \\
\text { infections }\end{array}$ \\
\hline Carbone et al. 2016 & Oral Secondary Syphilis \\
\hline Dickenson et al. 2000 & $\begin{array}{l}\text { Screening for syphilis in patients with carcinoma of the } \\
\text { tongue }\end{array}$ \\
\hline Ficarra et al. 2009 & $\begin{array}{l}\text { Syphilis: the renaissance of a old disease with oral } \\
\text { implications }\end{array}$ \\
\hline Gaul et al. 2015 & $\begin{array}{l}\text { A probable case of congenital syphilis from pre- } \\
\text { Columbian Austria }\end{array}$ \\
\hline Jones et al. 2012 & Three cases of oral syphilis - a overview \\
\hline Little et al.2005 & Syphilis: a update \\
\hline Minicucci et al. 2013 & $\begin{array}{l}\text { Oral manifestations of secondary syphilis in the elderly - } \\
\text { a timely reminder for dentists }\end{array}$ \\
\hline Paulo et al.2015 & Oral manifestations of oral syphilis \\
\hline Scott et al.2005 & $\begin{array}{l}\text { Oral syphilis-re-emergence of an old disease with oral } \\
\text { manifestations }\end{array}$ \\
\hline Siqueira et al. 2014 & $\begin{array}{l}\text { Diagnostic approaches in unsuspected oral lesions of } \\
\text { syphilis }\end{array}$ \\
\hline Strieder et al. 2015 & $\begin{array}{l}\text { Oral syphilis: report of three cases and characterization } \\
\text { of the inflammatory cells }\end{array}$ \\
\hline
\end{tabular}

Quadro 1: Resultado do levantamento na base de dados.

\section{- Manifestações orais da sífilis terciária}

O estágio terciário da sífilis representa uma das fases mais complicadas da infecção, podendo comprometer o sistema cardiovascular e levar o paciente a óbito ${ }^{2}$. Os pacientes podem apresentar aneurisma da aorta ascendente, hipertrofia ventricular esquerda e insuficiência cardíaca congestiva ${ }^{4}$. Uma das complicações mais sérias nessa fase é o envolvimento do sistema nervoso central, causando demência, psicose, paralisia generalizada podendo levar o paciente a óbito em pouco tempo ${ }^{2,6}$. Lesões granulomatosas, denominadas goma, frequentemente são vistas e podem afetar tecidos moles, ossos, pele e órgãos internos ${ }^{7}$. Na cavidade oral, é frequente a presença desses leões. As localizações mais frequentes são o palato duro e língua, embora também possa acometer palato mole, rebordo alveolar inferior e glândulas parótidas ${ }^{2}$. Essas lesões podem se coalescer e formar grandes lesões ulceradas e causar intensa destruição tecidual. Quando acometem palato duro, podem causar perfuração com comunicação da cavidade nasal com a cavidade oral $^{2,7,8}$. Quando a língua é envolvida pode formar um padrão lobulado, irregular e difuso denominado de glossite intersticial. As papilas podem se apresentar atróficas ou totalmente ausente devido a infecção profunda na região, formando um padrão chamado de glossite luética ${ }^{1,2,4,9,10}$. Na prática diária, a associação da história da doença com as manifestações clínicas e reatividade aos testes sorológicos treponêmicos e não treponêmicos é suficiente para o diagnóstico da sífilis ${ }^{13,16}$. Alguns pacientes afetados pela sífilis oral são submetidos a biópsias para fins de diagnóstico, e características histológicas da sífilis secundária oral, embora inespecíficas, podem ser altamente sugestivas da doença ${ }^{14}$.

\section{- Manifestações orais da sífilis congênita}

A transmissão da sífilis de mãe para filho também pode ocorrer e é denominada sífilis congênita. Nos últimos dez anos os casos de sífilis congênita aumentaram praticamente 5 vezes no mundo ${ }^{4}$. Os achados clínicos na sífilis congênita foram descritos inicialmente em 1858, por Jonathan Hutchinson ${ }^{2}$. Uma tríade de sinais patognomônicos são geralmente vistos e as características incluem dentes de hutchinson, ceratite ocular intersticial e surdez associadas ao oitavo par de nervos $\operatorname{cranianos}^{15}$. Nem sempre os três sinais são encontrados, sendo necessárias informações clínicas adicionais e a observações de outros sinais clínicos, como bossa frontal, maxila atrésica e palato ogival ${ }^{4}$. Os sinais clínicos podem se manifestar semanas após o nascimento, com prejuízo ao crescimento da criança. Fissuras cutâneas podem se desenvolver nos lábios das crianças, associadas a lesões vesiculobolhosas, erupções cutâneas maculapapulares, febre, icterícia, anemia, hepatoesplenomegalia e rinite $^{2,7,4}$. Os dentes de hutchinson apresentam diâmetro mesiodistal maior no terço médio e se afunilam ao se aproximar do face oclusal, lembrando a parte ativa de uma chave de fenda ${ }^{4}$. O Quadro 2 mostra um resumo das principais alterações bucais da sífilis, de acordo com o estágio de infecção e as principais localizações das lesões.

\begin{tabular}{|l|l|l|}
\hline $\begin{array}{l}\text { Sífilis } \\
\text { Primária }\end{array}$ & $\begin{array}{l}\text { Ulcerações Inespecíficas } \\
\text { Proliferação vascular semelhante a a } \\
\text { granuloma piogênico }\end{array}$ & $\begin{array}{l}\text { Lábio } \\
\text { Língua } \\
\text { Palato } \\
\text { Gengivas } \\
\text { Amigdalas }\end{array}$ \\
\hline Sífilis \\
Secundária & $\begin{array}{l}\text { Placas mucosas esbranquiçadas } \\
\text { semelhantes a leucoplasia oral } \\
\text { Pápulas fendidas } \\
\text { Condiloma lata } \\
\text { Lesões orais maculopapulares }\end{array}$ & $\begin{array}{l}\text { Língua } \\
\text { Lábio } \\
\text { Mucosa Jugal } \\
\text { Palato }\end{array}$ \\
\hline $\begin{array}{l}\text { Sífilis } \\
\text { Terciária }\end{array}$ & $\begin{array}{l}\text { Inflamação granulomatosa (goma) } \\
\text { Glossite Intersticial } \\
\text { Glossite luética }\end{array}$ & $\begin{array}{l}\text { Palato } \\
\text { Língua }\end{array}$ \\
\hline $\begin{array}{l}\text { Sífilis } \\
\text { Congênita }\end{array}$ & $\begin{array}{l}\text { Incisivos de Hutchinson } \\
\text { Palates em amora andoado } \\
\text { Inflamações granulomatosas (gomas) } \\
\text { Glossite atrófica }\end{array}$ & $\begin{array}{l}\text { Dentes } \\
\text { Palato }\end{array}$ \\
\hline
\end{tabular}

Quadro 2: Resumo das principais manifestações orais da sífilis, de acordo com estágios e localizações das lesões.

- Diagnóstico da sífilis

Os testes sorológicos para diagnóstico da sífilis estão cada mais sensíveis e específicos. O VDRL (Venereal Disease Research Laboratory), RPR (rapid plasma reagin), e o FTA-ABS (fluorescent treponemal antibody absorption) são os mais utilizados ${ }^{14}$. Todos os testes sorológicos são altamente sensíveis para a sífilis no estágio secundário da infecção ${ }^{17}$. O VDRL apresenta níveis de sensibilidade de aproximadamente $100 \%{ }^{17}$. Alguns estudos têm sugerido os testes sorológicos, biopsia das lesões orais, quando se aplicar, somado às reações imuno-histoquímicas para detecção da espiroqueta $^{14}$. Entretanto, as lesões da sífilis não produzem características histológicas especificas ${ }^{14}$.

\section{CONCLUSÃO}

A sífilis é uma importante infecção 
bacteriana sistêmica e o cirurgião-dentista tem grande importância no diagnóstico devido às manifestações orais, que são frequentes, principalmente no estágio secundário. As manifestações clínicas podem mimetizar outras doenças; testes sorológicos, aliados a informações clínicas detalhadas devem ser realizados para o diagnóstico final da infecção.

\section{REFERÊNCIAS}

1. Leuci S, Martina S, Adamo D, Ruoppo E, Santarelli A, Sorrentino R et al. Oral Syphilis: a retrospective analysis of 12 cases and a review of literature. Oral diseases. 2013;19(8):738-46.

2. Leão JC, Gueiros LAM, Porter SR. Oral manifestations of syphilis. Clinics. 2006;61(2):161-66.

3. Bruce AJ, Rogers RS 3rd. Oral manifestations of sexually transmitted diseases. Clin Dermatol. 2004;22(6):520-27.

4. Neville BW, Damm DD, Allan CM, Chi AC. Patologia Oral e Maxilofacial. 4.ed. Elsevier: Rio de Janeiro; 2016.

5. Kojima N, Klausner JD. An update on the global epidemiology of syphilis. Curr Epidemiol Rep. 2018;5(1):24-38.

6. World Health Organization. Global health sector strategy on sexually transmitted infections 20162021. 2016. The WHO's strategy for STI treatment.

7. Carbone PN, Capra GG, Nelson BL. Oral Secondary Syphilis. Head Neck Pathol. 2016; 10(2):206-8.

8. Dickenson AJ, Currie WJ, Avery BS. Screening for syphilis in patients with carcinoma of the tongue. $\mathrm{Br} \quad \mathrm{J}$ Oral Maxillofac Surg. 1995;33(5):319-20.

9. Ficarra G, Carlos R. Syphilis: the renaissance of a old disease with oral implications. Head Neck Pathol. 2009;3(3):195-206.

10.Gaul JS, Grossschimdt K, Gusenbauer C, Kanz F. A probable case of congenital syphilis from preColumbian Austria. Anthropol Anz. 2015; 72(4):451-72.

11.Jones L, Ong ELC, Okpokman A, Sloan P, Macleod I, Staines KS. Three cases of oral syphilis - an overview. Br Dental J. 2012; 212(10):477-80.

12.Little JW. Syphilis: a update. Oral Surg Oral Med Oral Pathol Oral Radiol Endod. 2005;100(1):3-9.

13.Minicucci EM, Vieira RA, Oliveira DT, Marques SA. Oral manifestations of secondary syphilis in the elderly - a timely reminder for dentists. Aust Dent J. 2013;58(3):368-70.

14.Paulo LF, Servato JP, Oliveira MT, Duriguetto AF Jr, Zanetta-Barbosa D. Oral manifestations of oral syphilis. Int J Infect Dis. 2015;35:40-2.
15. Scott CM, Flint SR. Oral syphilis-re-emergence of an old disease with oral manifestations. Int $\mathrm{J}$ Oral Maxillofac Surg. 2005;34(1):58-63.

16.Siqueira CS, Saturno JL, Sousa SCO, Silveira FR. Diagnostic approaches in unsuspected oral lesions of syphilis. Int J Oral Maxillofac Surg. 2014; 43(12):1436-40.

17.Strieder LR, Léon JE, Carvalho YR, Kaminagakura E. Oral syphilis: report of three cases and characterization of the inflammatory cells. Annals of Diagnostic Pathology. 2015;19: 76-80.

\section{CONFLITO DE INTERESSES}

Os autores declaram não haver conflitos de interesse.

\section{AUTOR PARA CORRESPONDENCIA}

Erison Santana Santos

erisonsantosupe@hotmail.com

Submetido em 09/10/2018

Aceito em 06/02/2019 